\title{
Fixed point theorem for defuzzification and its application in decision making units
}

\author{
Elmira Abbasi ${ }^{1}$, Rahim Saneifard ${ }^{2}$ and Ercan Celik ${ }^{1}$ \\ ${ }^{1}$ Ataturk University Faculty of Science, Department of Mathematics, Erzurum, Turkey \\ ${ }^{2}$ Islamic Azad University Faculty of Science, Department of Mathematics, Oroumieh, Iran
}

Received: 12 September 2017, Accepted: 28 September 2017

Published online: 19 June 2019.

\begin{abstract}
In this article the author discusses the problem of defuzzification by minimizing the weighted distance between the two fuzzy quantities. Also, this study obtains the nearest point with respect to a fuzzy number showing that this point is unique and relative to the weighted distance. Through applying this point, this method is presented for effectively ranking of the fuzzy numbers and their images to deal with and overcome the deficiencies of the previous techniques. Finally, several numerical examples following the procedure indicate the ranking results are valid.
\end{abstract}

Keywords: Fuzzy number, defuzzification, ranking, weighted distance, weighted point.

\section{Introduction}

Representing fuzzy numbers by proper intervals is of a great importance. An interval representation of a fuzzy number may contain numerous uses and applications. Through applying such a representation, it is possible to apply some results in fuzzy number approaches derived in the field of interval number analysis. For example, it is applicable to a comparison of fuzzy numbers by using the order relations defined on the set of interval numbers. Various authors in [7, 10] have considered the crisp approximation of fuzzy sets. They have proposed a rough theoretic definition of that crisp approximation, called the nearest interval approximation of a fuzzy set. On the other hand, a very different approach to crisp approximation of fuzzy sets was applied in [2]. They have proposed a rough theoretic definition of that crisp approximation, called the nearest ordinary set of a fuzzy set, and they have proposed the construction and establishment of such a set. They have made sense of a much more discrete fuzzy sets. Their approximation of the given fuzzy set is not unique. Thus, this article is not proposed to explain this method. Having reviewed the previous interval approximations, this paper renders a method to find the weighted interval approximation of a fuzzy number with the attainment of two conditions. Firstly, this interval is a continuous interval approximation operator. Secondly, the parametric distance between this interval and the approximated number is minimal.

Being put all this together, through applying this interval, we achieve the nearest weighted point approximation with respect to weighted distance and prove that this point is unique. The main purpose of this article is to show that this nearest weighted point can be used as a crisp approximation of a fuzzy number. Therefore, by this approximation, this research is proposed to describe a new method for ranking of the fuzzy numbers. Different parts of this paper is put together as follows: In the part 2, this article reiterates and restates some fundamental consequences on fuzzy numbers. In the part 3, a crisp set approximation of a fuzzy number is achieved. In this part, very important and useful remarks are rendered. In part 4, a crisp approximation of a fuzzy number is obtained. In part 5, a new method for the ranking of the 
fuzzy numbers is proposed. In part 6, a comparative explanation between the proposed project in this article and previous studies in this regard is put forth.

\section{Preliminaries}

The basic definitions of a fuzzy number are given in $[3,6,8,9]$ as follows:

Definition 1. A fuzzy number $\boldsymbol{A}$ in parametric form is a pair $\left(L_{A}, R_{A}\right)$ offunctions $L_{A}(\alpha)$ and $R_{A}(\alpha)$ that $0 \leq \alpha \leq 1$, which satisfy the following requirements:

(1) $L_{A}(\alpha)$ is a bounded monotonic increasing left continuous function,

(2) $R_{A}(\alpha)$ is a bounded monotonic increasing left continuous function,

(3) $L_{A}(\alpha) R_{A}(\alpha), \quad 0 \leq \alpha \leq 1$.

Definition 2. The trapezoidal fuzzy number $A=\left(x_{0}, y_{0}, \sigma, \beta\right)$, with two defuzzifier $x_{0}, y_{0}$, and left

$$
A(x)=\left\{\begin{array}{l}
\frac{1}{\sigma}\left(x-x_{0}+\sigma\right), \text { if } x_{0}-\sigma \leq x \leq x_{0} \\
1, \text { if } x_{0} \leq x \leq y_{0} \\
\frac{1}{\beta}\left(y_{0}-x+\beta\right), \text { if } y_{0} \leq x \leq y_{0}+\beta \\
0, \text { otherwise }
\end{array}\right.
$$

If $x_{0}=y_{0}$, then $A=\left(x_{0}, \sigma, \beta\right)$ is called trapezoidal fuzzy number. The parametric from of triangular fuzzy number is $L_{A}(\alpha)$ $=x_{0}-\sigma+\sigma r, R_{A}(\alpha)=y_{0}+\beta-\beta r$,

Definition 3. For arbitrary fuzzy number $A \in F$ ( $F$ denotes the space of the fuzzy numbers) and $0 \leq \sigma \leq 1$, function $f: F \times$ $[\sigma, 1] \rightarrow F$ such that $f(A, \sigma)=\left(L_{A}, R_{A \sigma}\right)$ is called delta-vicinity of the fuzzy number $A$. Then there is

$$
L_{A \sigma}(\alpha)=\left\{\begin{array}{l}
L_{A}(\alpha), \text { if } \alpha \in[\delta, 1] \\
L_{A}(\delta), \text { if } \alpha \in[0, \delta]
\end{array}\right.
$$

and

$$
L_{A \delta}(\alpha)=\left\{\begin{array}{l}
R_{A}(\alpha), \text { if } \alpha \in[\delta, 1], \\
R_{A}(\delta), \text { if } \alpha \in[0, \delta]
\end{array}\right.
$$

If $A=\left(x_{0}, y_{0}, \sigma, \beta\right)$ is a trapezoidal fuzzy number, the parametric form of it is $A_{\delta}=\left(L_{A \sigma}, R_{A \sigma}\right)$ that is as follows :

$$
L_{A \delta}(\alpha)= \begin{cases}x_{0}-\delta+\sigma \alpha, & \text { if } \alpha \in(\delta, 1] \\ x_{0}-\delta+\sigma \delta, & \text { if } \alpha \in[0, \delta]\end{cases}
$$

and

$$
R_{A \delta}=\left\{\begin{array}{l}
y_{0}+\sigma-\beta \alpha, \text { if } \alpha \in(\delta, 1] \\
y_{0}+\beta+\sigma \delta, \text { if } \alpha \in[0, \delta]
\end{array}\right.
$$

Definition 4. The following values establish the weighted averaged representative and weighted width, respectively to the fuzzy number A:

$$
\begin{gathered}
I(A)=\int_{0}^{1}\left(c L_{A}(\alpha)+(1-c) R_{A}(\alpha)\right) p(\alpha) d a \\
D(A)=\int_{0}^{1}\left(R_{A}(\alpha)-L_{A}(\alpha)\right) p(\alpha) d a
\end{gathered}
$$

where $0 \leq c \leq 1$. 
Definition 5. [11]. For two arbitrary fuzzy numbers $A=\left(L_{A}, R_{A}\right)$ and $B=\left(L_{B}, R_{B}\right)$, the quantity

$$
\left.d_{P}(A, B)=\int_{0}^{1} f(\alpha)\left[L_{A}(\alpha)-L_{B}(\alpha)\right]^{2} d a+\int_{0}^{1} f(\alpha)\left[R_{A}(\alpha)-R_{B}(\alpha)\right]^{2} d a\right)^{\frac{1}{2}}
$$

here $f:[0,1] \rightarrow[0,1]$ is a bi-symmetrical (regular) weighted, is called the bi-symmetrical (regular) weighted distance between $A$ and $B$ based on $f$.

Definition 6. [7]. An operator I : $F \rightarrow($ set of closed intervals in $R)$ is called an interval approximation operator if for any $A \in F$ :

(a') $I(A) \subseteq \operatorname{supp}(A)$

(b') $\operatorname{core}(A) \subseteq I(A)$

(c') $\forall(\varepsilon>0) \exists(\sigma>0)$ s.t $d(A, B)<\sigma \Rightarrow d(I(A), I(B))<\varepsilon$, where $d: F \rightarrow[0,+\infty]$ denotes a metric defined in the family of all fuzzy numbers.

Definition 7. [7]. An interval approximation operator satisfying in condition $\left(c^{\prime}\right)$ for any $A, B \in F$ is called the continuous interval approximation operator.

\section{The nearest weighted interval of a Fuzzy number}

Various authors in [7, 12] have considered the crisp approximation of the fuzzy sets. They have proposed a rough theoretic definition of that crisp approximation which was titled as the nearest ordinary set and the nearest interval approximation of a fuzzy set. In this part, the researchers of this article propose another approximation titled as the weighted interval value approximation. Let $A=\left(L_{A}, R_{A}\right)$ be an arbitrary fuzzy number. This article is proposed to make sense of a closed interval $C_{d p}(A)=\left[L_{C}, R_{C}\right]$, which is the weighted interval to $A$ with respect to the metric $d_{p}$. Therefore, this article has to minimize

$$
D_{p}\left(A, C_{d p}(A)\right)=\left(\int_{0}^{1} f(\alpha)\left[\left(L_{A}(\alpha)-L_{C}\right)^{2}+\left(R_{A}(\alpha)-R_{C}\right)^{2}\right] d a\right)^{\frac{1}{2}},
$$

with respect to $I_{L}$ and $I_{R}$. In order to minimize $d_{p}$ it suffices to minimize

$$
\bar{D}_{p}\left(L_{C}, R_{C}\right)=d_{p}^{2}\left(L_{C}, R_{C}\right)
$$

Clearly, the parameters $L_{C}$ and $R_{C}$ which minimize Eq. (4) must satisfy in $\nabla \bar{D}_{p}\left(L_{C}, R_{C}\right)=\left(\frac{\partial \bar{D}_{P}}{\partial L_{C}}, \frac{\partial \bar{D}_{P}}{\partial R_{C}}\right)=0$ Therefore, this article has the following equations :

$$
\left\{\begin{array}{l}
\left.\frac{\partial \bar{D}_{P}\left(L_{C}, R_{C}\right)}{\partial L_{C}}=-2 \int_{0}^{1} f(\alpha)\left[R_{A}(\alpha)-L_{C}\right)\right] d \alpha=0 \\
\left.\frac{\partial \bar{D}_{P}\left(L_{C}, R_{C}\right)}{\partial R_{C}}=-2 \int_{0}^{1} f(\alpha)\left[L_{A}(\alpha)-R_{C}\right)\right] d \alpha=0
\end{array}\right.
$$

The parameters IL associated with the left bound and $I_{R}$ associated with the right bound of the nearest weighted interval can be found through applying Eq. (5) as follows:

$$
\left\{\begin{array}{l}
L_{C}=\int_{0}^{1} f(\alpha) L_{A}(\alpha) d \alpha \\
R_{C}=\int_{0}^{1} f(\alpha) R_{A}(\alpha) d \alpha
\end{array}\right.
$$


Remark. Since $\operatorname{det}\left[\begin{array}{ll}\frac{\partial \bar{D}_{P}^{2}\left(L_{C}, R_{C}\right)}{\partial L_{C}^{2}} & \frac{\partial \bar{D}_{P}^{2}\left(L_{C}, R_{C}\right)}{\partial R_{C} \partial L_{C}} \\ \frac{\partial \bar{D}_{P}^{2}\left(L_{C}, R_{C}\right)}{\partial L_{C} \partial R_{C}} & \frac{\partial \bar{D}_{P}^{2}\left(L_{C}, R_{C}\right)}{\partial R_{C}^{2}}\end{array}\right]=\operatorname{det}\left[\begin{array}{ll}1 & 0 \\ 0 & 1\end{array}\right]=1>0$, therefore $L_{C}$ and $R_{C}$ given by (6), $\operatorname{minimize}\left(d_{p}\left(A, C_{d_{p}}(A)\right)\right.$. Therefor, the interval

$$
C_{d_{p}}(a)=\left[\int_{0}^{1} f(\alpha) L_{A}(\alpha) d \alpha, \int_{0}^{1} f(\alpha) R_{A}(\alpha) d \alpha\right]
$$

is the nearest weighted interval approximation of fuzzy number $A$ with respect $d_{p}$. Now let's take into consideration that this article aims at approximating a fuzzy number by a crisp interval. Given this, the researchers have to use an operator $C_{d_{p}}$ which transforms fuzzy numbers into the family of closed intervals on the real line.

Lemma 1. [10]. $\left(2 \int_{0}^{1} f(\alpha) g(\alpha) d \alpha\right)^{2} \leq 2 \int_{0}^{1} f(\alpha) g^{2}(\alpha) d \alpha$.

Theorem 1. [10]. The operator $C_{d_{p}}$ is an interval approximation operator, i.e. $C_{d_{p}}$ is a continuous interval approximation operator.

\section{The nearest weighted point of a Fuzzy number}

Let $A=\left(L_{A}, R_{A}\right)$ be an arbitrary fuzzy number and $C_{d_{p}}(A)=\left[L_{C}, R_{C}\right]$ be the nearest weighted interval of it. If $L_{C}=R_{C}$, then $C_{d_{p}}(A)=\left[L_{C}, R_{C}\right]=N_{P}(A)$, is the nearest weighted point approximation to the fuzzy number $A$, and It's unique. The value of $N_{P}(A)$ is as follows :

$$
N_{P}(A)=\int_{0}^{1} f(\alpha)\left(L_{A}(\alpha)+R_{A}(\alpha)\right) d \alpha
$$

The above-stated equation introduces in the following Theorem.

Theorem 2. Let $\boldsymbol{A}=\left(L_{A^{\prime}}, R_{A}\right)$ be a fuzzy number and $f(\alpha)$ be a bi symmetrical weighted function. Then $N_{P}(A)$ is the nearest weighted point to the fuzzy number $A$.

Proof. For the proof of Theorem, It is adequate that we replace $L_{C}=R_{C}=N_{P}(A)$ in (4) and then minimize function $\bar{D}_{P}\left(A, N_{P}(A)\right)=d_{P}^{2}\left(A, N_{P}(A)\right)$ with respect to $N_{P}(\mathrm{~A})$. Thus this article has to minimize

$$
\left.\bar{D}_{P}\left(A, N_{P}(A)\right)=\left(\int_{0}^{1} f(\alpha) L_{A}(\alpha)-N_{P}(A)\right)^{2}+\left(R_{A}(\alpha)-N_{P}(A)\right)^{2}\right) d \alpha
$$

with respect to $N_{P}(A)$. It is clear that the parameter $N_{P}(A)$ which minimizes Eq. (8) must satisfy in $\nabla \bar{D}_{P}\left(A, N_{P}(A)\right)=$ $\left(\frac{\partial \bar{D}_{P}}{\partial N_{P}(A)}\right)=0$. Therefore, this article has:

$$
\left.\frac{\partial \bar{D}_{P}\left(A, N_{P}(A)\right)}{\partial N_{P}(A)}=-2 \int_{0}^{1} f(\alpha)\left[L_{A}(\alpha)-N_{P}(A)\right) N_{P}+R_{A}(\alpha)-N_{P}(A)\right] d \alpha=0
$$

The solution is

$$
N_{P}(A)=\int_{0}^{1} f(\alpha)\left(c L_{A}(\alpha)+(1+c) R_{A}(\alpha)\right) d \alpha
$$

Since $\frac{\partial \bar{D}_{P}}{\partial N_{P}^{2}}=2>0$, therefore $N_{P}(\mathrm{~A})$ actually minimize $\bar{D}_{P}\left(\mathrm{~A}, N_{P}(\mathrm{~A})\right)$ and simultaneously minimize

$$
d_{p}\left(A, N_{P}(A)\right)
$$

Theorem 3. The nearest weighted point approximation to a given fuzzy number A is unique. 
Proof. To demonstrate the uniqueness of the operator $N_{P}(\mathrm{~A})$, we show that for any $\mathrm{C} \in R, \bar{D}_{P}\left(\left(A, N_{P}(A)\right) \leq \bar{D}_{P}(A, C)\right.$ holds. We can write

$$
\begin{aligned}
\bar{D}_{P}(A, C)= & \left.\int_{0}^{1}\left((I(A)-C)^{2}\right)+(D(A)-C)^{2}\right) f(\alpha) \cdot d \alpha \\
= & \int_{0}^{1}\left(\left(I(A)+N_{P}(A)-N_{P}(A)-C\right)^{2}\left(D(A)+N_{P}(A)-C\right)^{2} f(\alpha) \cdot d a\right. \\
= & \int_{0}^{1}\left(\left(I(A)-N_{P}(A)\right)^{2}+\left(D(A)-N_{P}(A)\right)^{2} \cdot d \alpha+\int_{0}^{1}\left(\left(N_{P}(A)-\left(N_{P}(A)-C\right)^{2}\right) f(\alpha) \cdot d \alpha\right.\right. \\
& +2\left(N_{P}(A)-C\right) \int_{0}^{1}\left(\left(I(A)-N_{P}(A)\right)+\left(D(A)-N_{P}(A)\right)\right) f(\alpha) d \alpha
\end{aligned}
$$

The last sentence of the above diction is zero, hence

$$
\bar{D}_{P}(A, C)=\bar{D}_{P}\left(A, N_{P}(A)\right)+2\left(N_{P}(A)-C\right)^{2}
$$

Consequently $\bar{D}_{P}(A, C)-\bar{D}_{P}\left(A, N_{P}(A)\right)=2\left(N_{P}(A)-C\right)^{2} \geq 0$; then we have $\bar{D}_{P}(A, C) \geq \bar{D}_{P}\left(A, N_{P}(A)\right)$, which completes the proof of theorem.

Remark. Let A and B be two fuzzy numbers and $\lambda$ and $\mu$ be positive numbers. Then we have

$$
N_{P}(\lambda A \pm \mu B)=\lambda N_{P}(A) \pm \mu N_{P}(B) .
$$

Proof. Let us suppose for all $0 \leq \alpha \leq 1, A=\left(L_{A}(\alpha), R_{A}(\alpha)\right)$ and $B=\left(L_{B}(\alpha), R_{B}(\alpha)\right)$ and $f(\alpha)$ is a bi-symmetrical weighted function. Then,

$$
\lambda A \pm \mu B=\lambda\left(L_{A}(\alpha), R_{A}(\alpha)\right) \pm \mu\left(L_{B}(\alpha), R_{B}(\alpha)\right)=\left(\lambda L_{A}(\alpha) \pm \mu L_{B}(\alpha), \lambda R_{A}(\alpha) \pm \mu R_{B}(\alpha)\right.
$$

Then

$$
\begin{gathered}
N_{P}(\lambda A \pm \mu B)=\int_{0}^{1} f(\alpha)\left[\lambda L_{A}(\alpha) \pm \mu L_{B}(\alpha)+\lambda R_{A}(\alpha) \pm \mu R_{B}(\alpha)\right] d \alpha \\
=\lambda \int_{0}^{1} f(\alpha)\left[\lambda L_{A}(\alpha)+R_{A}(\alpha)\right] d \alpha \pm \mu \int_{0}^{1} f(\alpha)\left[L_{B}(\alpha)+\mu R_{B}(\alpha)\right] d \alpha=\lambda N_{P}(A) \pm \mu N_{P}(B) .
\end{gathered}
$$

\section{Ordering of the Fuzzy numbers by the nearest weighted point}

In this part, the researchers propose the ranking of the fuzzy numbers associated with the nearest weighted point approximation. Ever, the nearest weighted point is applicable as a crisp approximation of a fuzzy number, therefore, the resulting approximation is used to rank the fuzzy numbers. Thus, $N_{P}($.$) is used to rank the fuzzy numbers.$

Definition 8. Let $A, B \in F$ be two fuzzy numbers, and $N_{P}(A)$ and $N_{P}(B)$ are the nearest weighted point of them. Define the ranking of $A$ and $B$ by $N_{P}$ on $F$, i.e.

(1) $N_{P}(A)<N_{P}(B)$ if and only if $A \prec B$

(2) $N_{P}(A)>N_{P}(B)$ if and only if $A \succ B$

(3) $N_{P}(A)=N_{P}(B)$

if $N_{P}\left(A_{\delta}\right)<N_{P}\left(B_{\delta}\right)$ then $A \prec B$,

else if $N_{P}\left(A_{\delta}\right)>N_{P}\left(B_{\delta}\right)$ then, $A \succ B$ 
else $A \sim B$ via Theorem 1, the nearest weighted point approximation of the $\delta$ - vicinity $A$ is as follows:

$$
N_{P}\left(A_{\delta}\right)=\int_{0}^{1} f(\alpha)\left(L_{A \delta}(\alpha)+R_{A \delta}(\alpha)\right) d \alpha
$$

Then, this article formulates the order $>$ and $\underset{\sim}{<}$ as $\mathrm{A}>\mathrm{B}$ if and only if $\mathrm{A} \succ \mathrm{B}$ or $\mathrm{A} \sim \mathrm{B}, \mathrm{A} \underset{\sim}{\sim} \mathrm{B}$ if and only if $\mathrm{A} \prec \mathrm{B}$ or $\mathrm{A} \sim$ B. This article takes into consideration the following reasonable axioms that Wang and Kerre [13] proposed for the fuzzy quantities ranking.

Let $I$ be an ordering method and $S$ the set of fuzzy quantities for which the method $I$ can be applied and $F$ a finite subset of $S$. If $I$ is applied to the fuzzy quantities then $A, B$ in $F$ satisfy that $A$ has a higher ranking than $B$, that is $A \succ B$ by $I$ on $F$.

$A \sim B$ by $I$ on $F$, and $A \geq B$ by $I$ on $F$ are similarly interpreted. The following proposition demonstrates the reasonable properties of the ordering approach $I$. Let $S$ be the set of fuzzy quantities for which the nearest point method can be applied, and $F$ and $F^{\prime}$ are two arbitrary finite subsets of $S$. Then we have:

(a) For an arbitrary finite subset of $\mathrm{S}$ and $A \in A ; A \succ A$.

(b) For an arbitrary finite subset $\mathrm{A}$ of and $(A, B) \in A^{2} ; A \succ B$ and $B \succ A$ results in $A \sim B$.

(c) For an arbitrary finite subset $\mathrm{A}$ of and $(A, B, C) \in A^{3} ; A \succ B$ and produces .

(d) For an arbitrary finite subset of and $(A, B) \in A^{2}$; inf $\left.\sup p(A)\right\rangle \sup \sup p(B)$; gives.

(e) For an arbitrary finite subset $A$ of $S$ and concludes as $A \succ B$.

(f) Let and $S^{\prime}$ be two arbitrary finite sets of fuzzy quantities in which $C_{d_{p}}($.$) is applicable, where both \mathrm{A}$ and B belong to $S \cap S^{\prime}$. Thus, the ranking order by $C_{d_{p}}($.$) on \mathrm{S}^{\prime}$ iff by $C_{d_{p}}($.$) on is achieved.$

(g) Let $A, B, A+C$ and $B+C$ be elements of .If ,then by on $\{\mathrm{A}+\mathrm{C}, \mathrm{B}+\mathrm{C}\}$.

(h) Let $A, B, A+C$ and be elements of . If by $C_{d_{p}}($.$) on and B$, then $\mathrm{A}+C \succ B+C$ by $C_{d_{p}}($.$) on \{A+C, B+C\}$.

Remark. Ranking order $N_{P}$ has the axioms $A_{1}, A_{2}, \ldots, A_{6}$.

Proof. The proof is similar to [11].

Remark. If $A \prec B$, then $-A \succ-B$. Hence, this article can conclude the ranking order of the images of the fuzzy numbers.

\section{Examples}

In this part, we are to compare the proposed method with others [12].

\subsection{Example}

Consider the data used in [12], i.e., the three fuzzy numbers, $A=(5,6,7), B=(5.9,6,7)$ and $C=(6,6,7)$, as shown in Figure 1.

Given the Eq.(4.10) the ranking index values are achieved, i.e., $N_{P}(A)=6,6 N_{P}(B)=.15$ and $. N_{P}(C)=6.16$ Thus, the ranking order of fuzzy numbers is $A \prec B \prec C$. However, by Chu and Tsao's Approach [5], the ranking order is . Meanwhile, using the proposed CV index, the ranking order is $B \prec C \prec A$. From Fig. 1, It is obvious that the ranking results obtained by the existing approaches [4,5] are unreasonable and inconsistent. On the other hand, in [1], the ranking result is $C \prec B \prec A$, which is the same as the one obtained by the proposed method. However the approach rendered in this research is proposed as a much simpler one in the computation procedure. Based on the analysis results from [1], the ranking results of this effort and other approaches are listed in Table 1. 


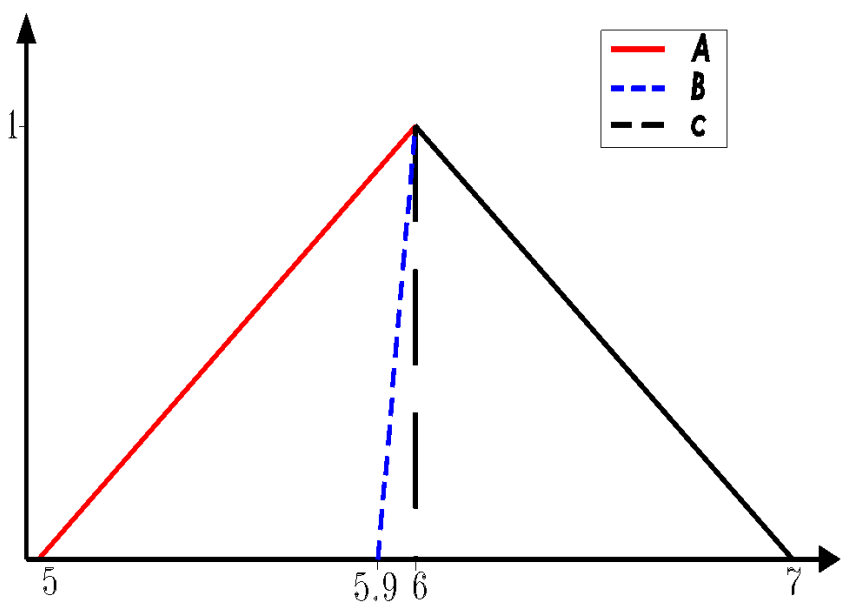

Fig. 1

Table 1

\begin{tabular}{|c|c|c|c|c|c|c|}
\hline Fuzzy number & New approach & $\begin{array}{c}\text { Sign Distance } \\
\text { with } \mathbf{~ = 1}\end{array}$ & $\begin{array}{c}\text { Sign Distance } \\
\text { with } \mathbf{p = 2}\end{array}$ & Chu-Tsao & Cheng Distance & index \\
\hline A & 6 & 6.12 & 8.52 & 3.000 & 6.021 & 0.028 \\
\hline B & 6.15 & 12.45 & 8.82 & 3.126 & 6.349 & 0.009 \\
\hline C & 6.16 & 12.50 & 8.85 & 3.085 & 6.351 & 0.008 \\
\hline Results & & & $A \prec B \prec C$ & $A \prec B \prec C$ & $A \prec B \prec C$ & $A \prec C \prec B$ \\
\hline
\end{tabular}

\subsection{Example}

Consider the data used in [12], i.e., the four fuzzy numbers, $\mathrm{A}=(-1,0,1), \mathrm{B}=(2.2,2.75,3.5), \mathrm{C}=(2,3,3.5)$ and $\mathrm{D}=(4,5,6)$. According to Eq.(4.10) the ranking index values are obtained, i.e., $N_{P}(A)=1, N_{P}(B)=1.63, N_{P}(C)=2.4$ and $N_{P}(D)=5$. The ranking order of the fuzzy numbers is $A \prec B \prec C \prec D$. But by the Z.-X. Wang method [14], the ranking order is $A \prec C \prec B \prec D$.

Which is unreasonable and this is another shortcoming of the Z.-X. Wang method. In fact, we can provide many examples like the one stated above.

\section{Conclusion}

In this paper, the authors proposed a defuzzification using minimizer of the weighted distance between two fuzzy numbers. Through the application of this defuzzification, we made sense of a new method for the ranking of the fuzzy numbers. Roughly, there is no in-depth distinction between our proposed method and the ones already proposed by other scholars. This method is capable of effectively ranking the various fuzzy numbers and their images.

\section{Competing interests}

The authors declare that they have no competing interests. 


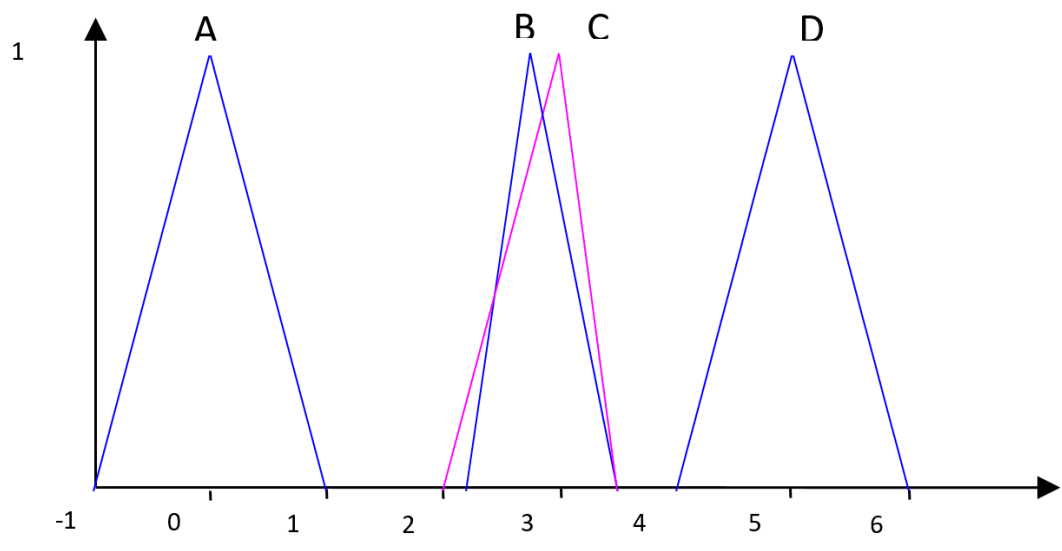

Fig. 2

\section{Authors' contributions}

All authors have contributed to all parts of the article. All authors read and approved the final manuscript.

\section{References}

[1] Abbasbandy, S., Asady, B., 2006. Ranking of fuzzy numbers by sign distance., Information Science.(176) 2405-2416.

[2] Chakrabarty, K., Biswas, R., Nanda, S., 1998. Nearest ordinary set of a fuzzy set: a rough theoretic construction, Bull., Polish Acad. Sci. (46) 105 - 114.

[3] Chu, H., Lee-K wang, H., 1994., Ranking fuzzy values with satisfication function, Fuzzy Sets and Systems. (64) 295-311.

[4] Cheng, C. H., 1999. Ranking alternatives with fuzzy weights using maximizing set and minimizing set, Fuzzy Sets and System. (105) 365-375.

[5] Chu, T., Tsao, C., 2002. Ranking fuzzy numbers with an area between the centroid point and original point, Comput. Math. Appl.( 43) 11-117.

[6] Dubois, D., Prade, H., 1987. The mean value of a fuzzy number, Fuzzy Sets and Systems. (24) 279-300.

[7] Grzegorzewski, P.,2002. Nearest interval approximation of a fuzzy number, Fuzzy Sets and Systems.(130) 321-330.

[8] Heilpern, S., 1992.The expected value of a fuzzy number, Fuzzy Sets and Systems.(47) 81-86.

[9] Kauffman, A., Gupta, M. M.,1991. Introduction to fuzzy arithmetic: Theory and Application, Van Nostrand Reinhold, New York.

[10] Saneifard, R., 2009. Ranking L-R fuzzy numbers with weighted averaging based on levels, International Journal of Industrial Mathematics. (2) 163-173.

[11] Saneifard, R.,2010. Defuzzification through a bi-symmetrical weighted function,Australian Jornal of .Basic and Application Sciences.(4) 4856-4865.

[12] Saneifard, R., 2009. A method for defuzzification by weighted distance , International Journal of Industrial Mathematics. (3) 209-217.

[13] Wang, X., Kerre, E. E., 2001. Reasonable properties for the ordering of fuzzy quantities (I), Fuzzy Sets and Systems.( 118) 378405.

[14] Wang, Z. X., Liu, Y. J., Fan, Z. P. \& Feng, B. (in press). Ranking LR fuzzy number based 395 on deviation degree. Information Sciences. 